\title{
Zur Klassifikation von Geschäftsmodellen im Market Space
}

Die Gestaltung digitaler Produkte und Dienstleistungen verlangt, mithilfe eines Geschäftsmodells sowohl Kooperationsformen (Business Webs) wie Nutzenpotenziale zu evaluieren. Dieser Beitrag erläutert die Komponenten webbasierter Geschäftsmodelle inklusive der Gestaltung der Geschäftsprozesse, Festlegung eines Preismodells und einer Sicherheitskonzeption. Anschließend werden die fünf Business Webs von Tapscott vorgestellt, die eine Klassifikation webbasierter Geschäftsmodelle im Market Space zulassen: Agora (freier Marktplatz), Aggregator, Integrator, Allianz (selbstorganisierte Gemeinschaft) und Distributor. Vergleich und Bewertung dieser Kooperationsformen werden ebenfalls vorgenommen und an konkreten Beispielen illustriert.

\section{Inhaltsübersicht}

1 Komponenten eines webbasierten Geschäftsmodells

2 Business Web vom Typ Agora

3 Business Web vom Typ Aggregator

4 Business Web vom Typ Integrator

5 Business Web vom Typ Allianz

6 Business Web vom Typ Distributor

7 Vergleich und Bewertung von Netzwerken

8 Ausblick

9 Literatur

\section{Komponenten eines webbasierten Geschäftsmodells}

Im Zuge der Entwicklung hin zu einer vernetzten Informationsgesellschaft kann eine Veränderung der Marktsysteme für die Unternehmen beobachtet werden [Bliemel et al. 2000; Meier \& Stormer 2005; Wirtz 2000]. Zum einen bleibt der physische Marktplatz (oft als Market Place bezeichnet) mit physischen Rohstoffen, Produkten und Ressourcen bestehen. Hier sind physische Wertschöpfungsprozesse notwendig, um materielle Güter zu beschaffen, zu entwickeln und zu verteilen. Durch die Entwicklung des Internets wird dieser physische Marktplatz ergänzt. Dabei spricht man von einem digitalen Marktraum (oder Market Space), um anzudeuten, dass nun zusätzlich digitale Produkte und Dienstleistungen in Netzwerken entwickelt und abgesetzt werden können.

Die Unternehmen müssen sich in einem Geschäftsmodell entscheiden, ob und wie der physische und der elektronische Marktraum bearbeitet werden [Bullinger \& Berres 2000; Meier 2001]. Die Unternehmensstrategie muss bzw. kann in den meisten Fällen beide Marktoptionen einbeziehen. Mit anderen Worten stellt sich die Grundsatzfrage, wie ein Erfolg versprechender Mix von materiellen und immateriellen Produktteilen oder Dienstleistungen festzulegen ist und wie die entsprechenden Geschäfte abgewickelt werden.

Ein webbasiertes Geschäftsmodell für den Market Space [Timmers 1999] verlangt die Klärung folgender Fragestellungen:

- Definition der Produkte und Dienstleistungen inklusive Digitalisierungsgrad: Es muss festgelegt werden, ob die bestehende Produktpalette durch digitale Informationsobjekte und Servicedienste ergänzt, abgelöst oder erweitert werden soll. Der Digitalisierungsgrad einzelner Produkte und Dienstleistungen ist abhängig von Markt und Akzeptanz der bestehenden oder angestrebten Kundschaft.

- Festlegung der Zielkunden, Zielkundensegmente und Absatzmärkte: Durch Analyse- 
arbeiten (z. B. Nutzung Data Warehouse und Data Mining) werden diejenigen Kunden und Kundensegmente evaluiert, die einen hohen Kundenwert besitzen. Zudem werden potenzielle Kundengruppen avisiert, die das Kundenkapital erhöhen und das Absprung- oder verlustrisiko vermindern.

- Evaluation und Auswahl des geeigneten Business Web: Business Webs [Tapscott et al. 2001] sind Netzwerke für Herstellung und Vertrieb digitaler Produkte und Dienstleistungen. Zielsetzung und Hauptmerkmale von Business Webs reichen von freien Marktplätzen mit verhandelbaren Waren und Dienstleistungen (Typ Agora, siehe Abschnitt 2) über hierarchisch straff organisierte Netzwerke (Typ Aggregator, Integrator oder Distributor, siehe Abschnitte 3, 4 und 6) bis zu selbstorganisierten und lose gekoppelten Gemeinschaften (Typ Allianz, siehe Abschnitt 5).

- Gestaltung der Geschäftsprozesse inklusive Distribution: Bei der Gestaltung der Geschäftsprozesse bzw. Leistungserstellungsprozesse wird festgelegt, welche Aktivitäten automatisch und welche manuell durchgeführt werden. Die Distributionslogistik und die Festlegung der Lieferantenkette (Supply Chain Management) sind ebenfalls bedeutend, können doch Online-, Offline- oder hybride Verteil- und Bezugsstrukturen gewählt werden. Zu diesem Entscheidungsbereich gehört auch die Organisation übergreifender Kooperationsprozesse im Falle virtualisierter Kooperationsnetzwerke mit einem eher partnerschaftlichen Grundverhältnis.

- Entwicklung eines Preismodells und Klärung der Zahlungsmodalitäten: Die originäre Produktion eines digitalen Gutes ist kostenintensiv, im Gegensatz dazu sind die Grenzkosten der Reproduktion, d.h. die Erstellung von Kopien, vernachlässigbar resp. gering [Shapiro \& Varian 1999]. Zudem wächst der generierte Nutzen mit der Anzahl der Konsumenten von Produkten und Dienstleistungen (Netzwerk- effekte). Neben der Preisbildung, -differenzierung und -bündelung gilt es, geeignete elektronische Zahlungsoptionen zu prüfen und bei Bedarf anzubieten (Electronic oder Mobile Payment).

- Erstellung einer Sicherheitskonzeption: Ein Risikoraster mit Chancen und Risiken elektronischer Geschäfte [Shaw et al. 2000] trägt dazu bei, vorhandene Ressourcen und Mittel abzuwägen und bei Bedarf Zusatzinvestitionen zu tätigen. Eine Sicherheitskonzeption muss umgesetzt werden, um Integrität, Verfügbarkeit und Vertraulichkeit elektronischer Geschäfte zu gewährleisten (vgl. Kryptografieverfahren und digitale Signatur z. B. in [Meier \& Stormer 2005]).

- Festlegung des Finanzierungs- bzw. Kapitalmodells: Das Finanzierungsmodell zeigt die Quellen auf, aus denen das zur Finanzierung der Unternehmenstätigkeit eingesetzte Kapital stammt. Das Erlösmodell beschreibt die Art, anhand welcher Bezugsgrößen und in welcher "Berechnungsformel " Erlöse entstehen. [Wirtz 2000] schlägt eine Klassifikation von Erlösformen vor, die Erlöse zum einen nach dem Akteur, von dem sie bezogen werden, in direkte und indirekte Erlöse, zum anderen nach den Preiskonditionen in transaktionsabhängige und transaktionsunabhängige Erlöse unterteilt.

Aufgrund der Erweiterung des physischen Marktplatzes um einen elektronischen Marktraum, der Globalisierung vieler Geschäftstätigkeiten, der steten Entwicklung hin zu einer Informationsgesellschaft sowie der gemachten Erfahrungen nach dem Platzen der Internetblase müssen die Unternehmen ihren eigenen Marktfokus überdenken [Schögel et al. 2002] und mögliche Chancen abwägen. Dabei hilft die Konzentration auf Kernkompetenzen und Netzwerkbildung (Aufbau geeigneter Business Webs) mit Erfolg versprechenden Partnern. Im Folgenden soll die Typologie der Business Webs nach [Tapscott 2001] vorgestellt und anhand von praktischen Beispielen illustriert werden. 
Weiterführende Geschäftsmodelle unter Nutzung von Web 2.o-Technologien werden in einem weiteren HMD-Beitrag von [Wirtz \& Ullrich 2008] in diesem Heft beschrieben. Die Praxisbeispiele setzen sich vor allem mit den Erfolgsfaktoren der Realisierung der einzelnen Geschäftsmodelle auseinander.

\section{Business Web vom Typ Agora}

Der Begriff Agora stammt aus der Antike und bezeichnete die damals abgehaltenen Volksversammlungen. Später nannte man den öffentlichen Versammlungsort, an dem mehr und mehr auch Handel betrieben wurde, selbst Agora.

Das Business Web oder B-Web Agora ist ein elektronischer Marktplatz, auf dem Käufer und Verkäufer zusammenkommen, um frei über die angebotenen Güter und deren Preise zu verhandeln. Wichtig bei der Agora ist der Prozess der dynamischen Preisfindung: Bei einer Agora gibt es keine Fixpreise, die Preise werden verhandelt. Eine Agora fördert demnach den Austausch von digitalen und materiellen Gütern und Dienstleistungen zu individuell vereinbarten Preisen.

In Abbildung 1 wird die Grundstruktur des B-Web Agora gezeigt: Anbieter oder Verkäufer, symbolisch durch Kreise charakterisiert, bieten ihre Waren und Dienstleistungen auf dem vir- tuellen Marktplatz an. Nachfrager oder Käufer, als Dreiecke dargestellt, informieren sich vorerst und verhandeln die Produktteile inklusive Nutzungsrechte und Preise individuell.

Auf einer Agora können unterschiedliche Leistungserbringer Produkte und Dienstleistungen anbieten und absetzen. Das Angebot ist vielfältig und nicht vorhersehbar, die Wertintegration bleibt jedoch bescheiden; die Anbieter profitieren vom "Fühlungsvorteil« und der erreichten Transparenz. Gut funktionierende Marktplätze nach dem Muster einer Agora zeigen sowohl für die Anbieter- wie für die Nachfragerseite Vorteile. Bei einer hohen Anzahl von Anbietern mit unterschiedlicher Anzahl von Produkten und Dienstleistungen profitieren die Käufer. Umgekehrt setzen viele Nachfrager mit unterschiedlichen Produkt- und Preisvorstellungen die Anbieter unter Druck.

Eine Agora bietet normalerweise differenzierte Informationen über alle Aspekte des Geschäfts. Gleichzeitig hält sie Marketing- und Vertriebskosten gering. Oft verleihen Schnäppchenjäger den Marktteilnehmern einen besonderen Reiz und bieten gesuchte Unterhaltung. Das Internet kann Agoren in interessante Treffpunkte mit effizienten Marktabläufen verwandeln. Waren oder Dienstleistungen, die bis dahin an lokalen Orten, Flohmärkten oder im

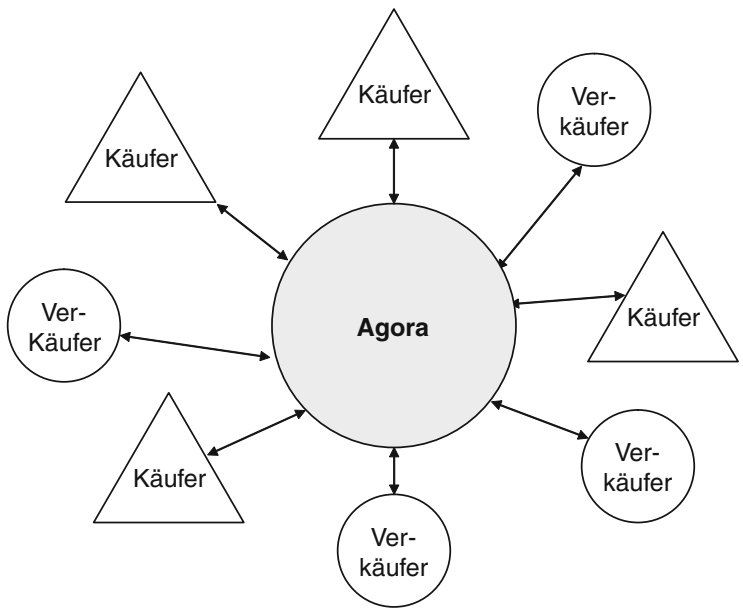

Abb. 1: Agora als offener Marktplatz mit dynamischer Preisfindung 
Kleinanzeigenteil von Tageszeitungen verkauft wurden, tauchen plötzlich in globalen Auktionen und Börsen auf.

Der zentrale Wert einer Agora liegt im Angebot einer vertrauensbildenden Plattform für den Austausch von Informationen, der Möglichkeit einer dynamischen Preisfindung und im Anstoß zu Leistungsabwicklungen. Solche Plattformen machen sich niedrige Transaktionskosten zunutze: Die Kunden, ob Anbieter oder Nachfrager, übernehmen den größeren Teil der Arbeit sowie weitgehend die Kosten und Risiken.

Plattformen vom Typ Agora zeigen für den Handel von materiellen und digitalen Gütern folgende Vorteile:

- Keine Lagerkosten: Die Anbieter lagern ihre Produkte selber.

- Minimale Marketingkosten: Die Anbieter beschreiben und illustrieren ihre Produkte auf der Plattform selbst.

- Reduzierte Vertriebskosten: Käufer und Anbieter regeln den Versand und die Bezahlung unter sich.

- Geringe Produkthaftung: Produkte werden versteigert; der Käufer trägt im Normalfall selbst das Risiko.

- Geringes finanzielles Risiko: Die Anbieter ermächtigen den Betreiber solcher Austauschplattformen zum Einzug einer Auktionsgebühr.

In einer Agora entwickeln sich die Kunden oder Kundengruppen oft zu einer Gemeinschaft (Community). Die Teilnehmer von Auktionen stimmen der Tatsache zu, dass Verhandlung, Preisfindung und Verteilung von Gütern als selbstorganisierender Prozess zu regeln sind.

Das weltweit bedeutendste Beispiel einer erfolgreichen Umsetzung dieser Form eines Business Web ist zweifellos eBay. Das im Jahr 1995 unter dem Namen auctionweb gegründete Unternehmen gilt vielen Nutzern und in der Sekundärliteratur als nahezu perfekte Realisierung eines idealen Marktes, da es eine hohe Anzahl von Anbietern und Nachfragern in ei- nem transparenten System vereinigt. eBay hat in seiner Geschichte eine bemerkenswerte Wandlung vom virtuellen Flohmarktbetreiber hin zu einer professionellen B2C-Plattform durchgemacht und sein Kerngeschäft "Marktplatz « durch die Integration des Bezahldienstes PayPal (siehe z. B. [Meier \& Stormer 2005]) und die Internettelefonie Skype erweitert. Die Umsatz- und Gewinnentwicklung verläuft positiv, obwohl eBay die Angebotsgebühren und Provisionen erhöht hat.

Bemerkenswert ist, dass eBay zu einer Plattform von neuen Unternehmensgründungen mit differenzierten Geschäftsmodellen geworden ist und ständig an der Ausdifferenzierung arbeitet. So sind unterschiedliche Klassen von zertifizierten Anbietern wie der PowerSeller, die eBay-Verkaufsagentur oder der eBay-Shop entstanden, die allesamt auf dem wesentlichen Gut der Verkäuferbewertung bzw. entsprechender Reputationssysteme (vgl. Beitrag von [Peters \& Reitzenstein 2008] in diesem Heft) beruhen und damit einen Wert darstellen. Allerdings unterliegen diese Reputationssysteme laufender Anpassung: So ist in eBay die Möglichkeit der Käuferbewertung abgeschafft worden, um »Rachebewertungen « schlecht bewerteter Verkäufer zu verhindern. Zusätzliche Dienste von eBay für die Anbietergruppen werden professionell erarbeitet, z. B. bis hin zu Seminarangeboten in großen Städten für potenzielle Einsteiger. Als erfolgskritisch gelten am Beispiel eBay die frühe Marktpräsenz der Plattform, die Medienpräsenz und Marktaufmerksamkeit, die dauerhafte Diversifikation und Investition in ergänzende Dienstleistungen sowie die hohe Usability der Plattform.

\section{Business Web vom Typ Aggregator}

Ein B-Web vom Typ Aggregator ist ein digitaler Supermarkt: Er wählt geeignete Produkte und Dienstleistungen unterschiedlicher Hersteller aus, entscheidet über die entsprechenden Marktsegmente, legt Preise fest und kontrolliert die Abwicklung. 


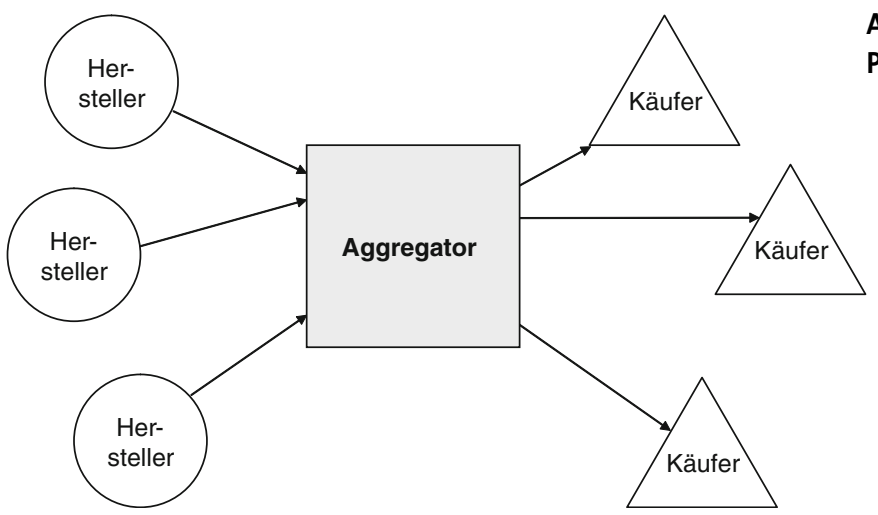

Abb. 2: Aggregator kombiniert Produkte und diktiert Preise

In Abbildung 2 ist die Konstellation eines Aggregators abstrakt dargestellt. Ein einzelnes Unternehmen eines solchen B-Web kontrolliert hierarchisch mehrere Hersteller. Der Aggregator kauft Produkte und Dienstleistungen nach eigenem Ermessen und legt die Einkaufspreise weitgehend selbst fest. Für das gewählte Sortiment bestimmt er die Verkaufspreise und Rabatte. Zudem kontrolliert er Absatz und Verteilung der Waren.

Aggregatoren übernehmen eine Vermittlerfunktion zwischen Herstellern und Kunden. Normalerweise bieten sie eine große Auswahl von Produkten und Diensten an, jedoch ohne oder mit lediglich minimaler Wertintegration (siehe dazu die Wertschöpfungskette des B-Web-Typs Integrator im folgenden Abschnitt).

Durch Marktvolumen und Marktmacht können Aggregatoren ihre Transaktionskosten senken, vor allem bei der Nutzung der Internettechnologien und entsprechender digitaler Agenten. Der digitale Supermarkt kann weitgehend mithilfe intelligenter Softwareagenten betrieben werden. Einfache Agenten beraten die Käufer, suchen und bewerten die gewünschten Produkte im eigenen Supermarkt oder direkt bei den Anbietern. Intelligente Agenten helfen den Kunden, ihre Wünsche einzugrenzen und aus der Vielfalt von Angeboten eine attraktive Kombination auszusuchen. Im Einzelfall können Softwareagenten über Werte- mix, Qualitätsanforderungen, Preis, Lieferungsbedingungen und Zahlungsmodalitäten verhandeln.

Zusammenfassend ergeben sich bei den Aggregatoren folgende Vorteile:

- Große Verhandlungsmacht: Der Aggregator wählt die Produkte aus und bestimmt die Preiskonditionen.

- Einsatz digitaler Berater: Softwareagenten unterstützen Such- und Vergleichvorgänge und beraten den Kunden.

- Unabhängige Produktbewertung: Vor- und Nachteile von Produkten werden von den Kunden erfasst und durch den Aggregator als Entscheidungshilfe publiziert.

- Stimulierung des Verkaufs: Im digitalen Supermarkt lassen sich Produkte bündeln und Cross-Selling-Maßnahmen realisieren.

- Kunde spart Versandkosten: Durch Skaleneffekte und tiefe Transaktionskosten kann der Aggregator Anreize schaffen.

Aggregatoren gibt es sowohl im B2B- wie im $\mathrm{B}_{2} \mathrm{C}$-Bereich. Neben Konsumgütern lassen sich digitale Produkte, z. B. Finanz- und Versicherungsdienstleistungen, mit Aggregatoren vertreiben.

Das bekannteste Beispiel für diese Form eines Business Web ist Amazon. Dieses 1995 gegründete, 1997 an die Börse gegangene Unternehmen bietet neben den anfänglich im Schwerpunkt gelegenen Medienprodukten Buch, 
Musik, CDs und DVDs eine Vielzahl von Produkten (Spielwaren, Elektronik, Haus- und Gartengegenstände, Sport- und Freizeitgüter) an und gilt als größter Online-Einzelhändler.

Geld verdient Amazon als virtueller Supermarkt mit teilweise hohen Margen, vor allem im Elektronik- und Spielzeugbereich. Zudem offeriert Amazon automatisierte Transaktionsund CRM-Dienstleistungen. Der Aggregator Amazon integriert die Käufer als Content-Lieferanten, z. B. bei der Abgabe von Rezensionen für Buchangebote. Er bietet eine Reihe von Zusatzfunktionen an, vor allem die Personalisierung des Zugangs und kontextgetriebene Angebote aufgrund ausgewerteter Nutzungs- und Kaufprofile. Zusätzlich offeriert Amazon individualisierte Benachrichtigungsdienste.

Amazon beherrscht die Logistik (z. B. Auslieferung häufig noch am gleichen Tag), die Möglichkeit der Rückverfolgung des Lieferprozesses im Web und einfache sowie vertrauenswürdige Bezahlfunktionen. Der Versand ist ab einem niedrigen Mindestwarenwert kostenlos. Als wesentliches Erfolgskriterium gilt wie bei eBay die große Marktpräsenz aufgrund des frühen Markteintritts, die Logistik, das individualisierte Kundenmanagement und nicht zuletzt die Vertrauenswürdigkeit des Bezahlverfahrens und die Zuverlässigkeit der Lieferung [Götze 2005]. Umsatz und Gewinn entwickeln sich in der jüngsten Vergangenheit gut, wobei gerade das
Wachstum in Deutschland, Großbritannien, Japan und China stark ist.

\section{Business Web vom Typ Integrator}

Ein Business Web vom Typ Integrator ist eine Wertschöpfungskette mit allen Komponenten von der Spezifikation, Produktion und Lieferung bis zum Support der vom Kunden gewünschten Produkte oder Dienstleistungen. Ein Integrator produziert selbst keine Dienstleistungen und Produktkomponenten, sondern wirkt als Kontextanbieter. Als solcher integriert er die Wertbeiträge verschiedener Inhaltsanbieter wie externer Entwickler, Teilelieferanten, Händler, Lösungsintegratoren, Betreiber und anderer Partner. Mit anderen Worten kontrolliert der Integrator die Gestaltung des Produkts resp. der Dienstleistung und lenkt die Schritte zur Wertintegration.

In Abbildung 3 ist das Grundprinzip eines Integrators veranschaulicht: Unterschiedliche Hersteller mit unterschiedlichen Fähigkeiten und Dienstleistungen werden zu Wertschöpfungsketten zusammengefasst und vom Integrator geführt. Der Anstoß einer solchen Wertschöpfungskette wird von Kunden gegeben, die eine individuelle und meist komplexe Lösung mit eventuell großem Investitionsvolumen anstreben. Da ein einzelner Hersteller die Lösung nicht optimal anbieten kann oder will, übernimmt der Integrator diese Verantwortung; oft

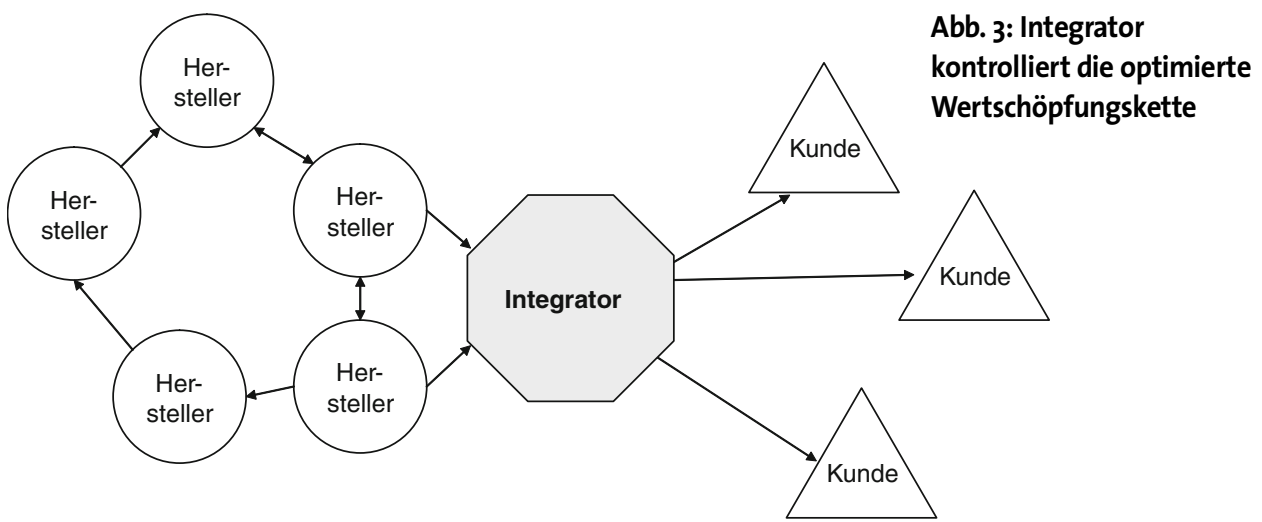


tritt er als Generalunternehmer mit entsprechender Haftung auf. Dies wiederum zwingt den Integrator, die Inhaltsanbieter in eine optimierte Lieferantenbeziehung einzubinden und den Planungs-, Entwicklungs-, Installationsund Serviceprozess kompetent zu steuern (vgl. Supply Chain Management).

Ein Integrator hat zum Ziel, die Wertschöpfungskette nachfrageorientiert zu gestalten. Mit anderen Worten schafft man ein Angebot erst auf eine Nachfrage hin. Ein Kundenbedürfnis löst also den Aufbau einer eventuell individuell zugeschnittenen Lieferantenkette aus.

Bei einer Wertschöpfungskette denkt man normalerweise an eine Massenproduktion oder Routineproduktion mit großer Logistik und Lagerhaltung. Wertschöpfungsketten vom Typ Integrator können hingegen die Werkstattfertigung unterstützen, z. B., es werden maßgefertigte Netzwerke für kundenspezifische Probleme entwickelt.

Eine Werkstattfertigung unterscheidet sich wesentlich von der Massenproduktion: Erstens sind die in einer Werkstattfertigung notwendigen Aktivitäten keine Routinevorgänge, sie werden aufgrund des Kundenproblems geplant und durchgeführt. Zudem folgt die Werkstatt nicht der Logik "Make and Sell«, sondern die Herstellung der Ware wird erst begonnen, wenn sie schon verkauft ist. Eine Werkstattfertigung orientiert sich somit an der Nachfrage und produziert "on Demand«. Der auftraggebende Kunde setzt die Wertschöpfungskette in Gang. Schließlich ist der Kunde einer Werkstattfertigung an der Konzeption und von Fall zu Fall auch an der Umsetzung einer Lösung mitbeteiligt.

Inhaltsanbieter in einem B-Web vom Typ Integrator sind oft verteilt und spezialisiert. Der Erfolg eines Integrators hängt demnach von guter Planung und Koordination der unterschiedlichen Partner ab. Dabei spielt das Projektmanagement eine wichtige Rolle, inklusive der Nutzung von Wissensressourcen.
Die Nutzenvorteile bei einem B-Web vom Typ Integrator lassen sich wie folgt zusammenfassen:

- Kundenlösung: Der Kundenauftrag steht am Anfang, erste Teilzahlungen erfolgen bei der Auftragserteilung.

- Generalunternehmer: Der Integrator übernimmt die Gesamtverantwortung für den Kundenauftrag.

- Bildung einer Wertschöpfungskette: Die Lieferantenauswahl samt der Vernetzung sowie entsprechende Verhandlungen werden vom Integrator durchgeführt.

- Werkstattfertigung anstelle Routinefertigung: Es werden auf den Kunden zugeschnittene Komponenten gefertigt.

- Projekt- und Methodenwissen: Der Integrator beherrscht Projektmanagement und Wissensverarbeitung.

In der digitalen Wirtschaft konkurrieren die besten Wertschöpfungsketten sowohl im Hinblick auf Kosten wie Differenzierung. Sie müssen kundenorientierte Lösungen suchen und anstelle von Massenwaren servicegestützte und individuelle Fertigung anbieten.

Ein bekanntes Beispiel eines Business Web vom Typ Integrator ist Cisco als Ausrüster von Telekommunikationsnetzen und -komponenten. Cisco verbindet in der Wertschöpfungskette unterschiedliche Unternehmen, so z. B. Halbleiterhersteller, Händler von Komponententeilen, Logistikunternehmen und Systemintegratoren.

Ein vom Fraunhofer IAO begleitetes Beispiel eines Business Web vom Typ Integrator mit nur teilweise digitalisierten Leistungserstellungsprozessen ist in der Strickindustrie in Oberitalien angesiedelt und reagiert dort auf die Markt- und Produktionssituation einer Vielzahl spezialisierter Textil- bzw. Strickwarenhersteller [Bullinger 1998]. Die Unternehmen der Wertschöpfungskette verfügen über eine große Spezialisierung, qualifizierte Fachkräfte und eine lange Tradition. Sie waren alleine auf sich ge- 
stellt und nicht in der Lage, die notwendigen Kontakte und Vertriebsbeziehungen zu den großen Bekleidungsherstellern bzw. Einkäufern der Modeindustrie in Mailand aufzubauen und zu pflegen. Dort hat eine privatwirtschaftliche, auf Mitgliederbeiträgen basierte Agentur diese Mittlerfunktion übernommen, die Kontakte geknüpft und ganze Kollektionen in Absprache mit den Auftraggebern zusammengestellt. Erst nach der Spezifikation und über Subvergabe an die vielen Einzelunternehmen wird neuerdings produziert und geliefert. Somit führt die Mittleragentur die gesamte Wertschöpfungskette, stellt die Teillieferanten zusammen und ist für die Abnahme verantwortlich. Dabei kommt dieser Agentur teilweise die Rolle eines gerechten Zuteilers von Aufträgen zu, um die Auslastungsschwankungen bei den Einzellieferanten über die Zeit hinweg zum Ausgleich zu bringen. Zusätzlich sorgt die Agentur in begrenztem Ausmaß für die notwendige Information und Weiterbildung der Lieferanten. Erfolgsgeheimnis sind die Akzeptanz des Mittlers bei Lieferanten wie Auftraggebern, die gute Kenntnis der individuellen Stärken und Schwächen der Lieferanten, deren Bündelung und damit eine Marktmacht gewinnende Funktion. Die Koordination und Kooperation wird durch Kollaborationssysteme, grafische Darstell ungsformen der Entwürfe und datenbankbasierte Verfahren unterstützt.

\section{Business Web vom Typ Allianz}

Allianzen als Business Web sind lose gekoppelte und selbstorganisierte Partnernetze (oft auch Gemeinschaften genannt), die eine gemeinsame Zielsetzung verfolgen. Die einzelnen Partner bringen ihr spezifisches Know-how ein und beteiligen sich gleichzeitig an der Lösungsentwicklung. Sie sind und bleiben unabhängig und versuchen, fehlende Kompetenzen durch Gewinnung geeigneter Netzwerkpartner wettzumachen.

In Abbildung 4 ist eine Allianz als Business Web illustriert. Die Mitglieder dieses Partnernetzes treten in Doppelfunktion auf: Sie haben ein Bedürfnis und suchen nach einer Lösung als Nachfrager oder Consumer (grafisch durch Dreiecke ausgedrückt), gleichzeitig beteiligen sie sich an der Lösungsentwicklung als Hersteller oder Producer (grafisch durch Kreise angedeutet). Mit der Wortschöpfung "Prosumer" wird die Doppelfunktion als Producer und Consumer untermauert.

Ein B-Web vom Typ Allianz ist ein dynamisches Gebilde gleichberechtigter Partner, das sich bei wechselnden Herausforderungen jeweils neu organisiert und weiterentwickelt. Dabei tritt kein Partner dominant auf und kontrolliert das Partnernetz. Vielmehr wird versucht, mit wenigen Verhaltensregeln das lose gekoppelte Partnernetz zusammenzuhalten.

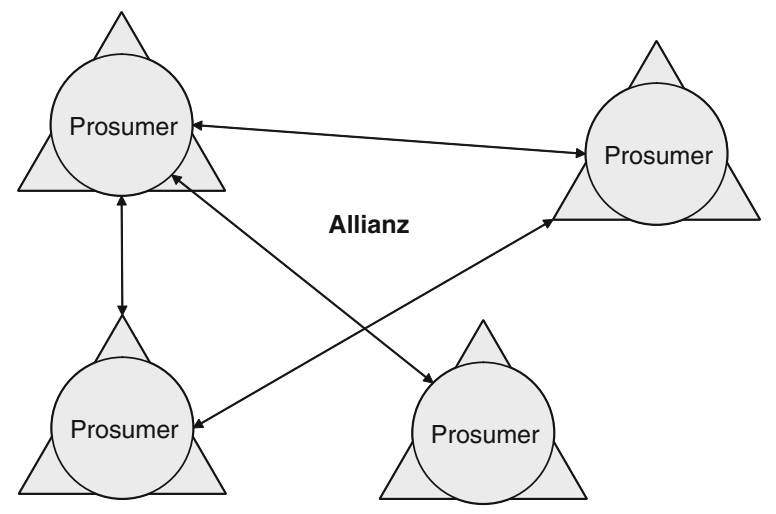

Abb. 4: Das B-Web Allianz bildet einen Wertschöpfungsraum 
B-Webs vom Typ Allianz entstehen auf freiwilliger Basis, motiviert durch ein gemeinsames Bedürfnis. Am Anfang steht oft eine kreative Idee, die gemeinsam mit weiteren Partnern umgesetzt werden soll. Gegenseitiger Respekt, immaterielle Werte und unkonventionelle Ideen sind die Treiber solcher Partnernetzwerke. Allianzen zeichnen sich durch eine hohe Wertintegration aus; man spricht in diesem Zusammenhang von einem Wertschöpfungsraum.

In vielen Fällen sind Allianzen zeitlich befristet. Sie entstehen, indem Kleinunternehmen oder Einzelpersonen sich zusammenfinden und eine gemeinsame lösung entwickeln. Ist die Lösung gefunden, wird sie verbreitet und weitergegeben, im Normalfall gebührenfrei.

Die Bildung von Allianzen ist vielfältig. Neben Netzwerken zur Entwicklung von OpenSource-Softwarepaketen gibt es soziale Gemeinschaften, spezifische Diskussions- und Hilfeforen, Netzwerke für freischaffende Künstler, Expertengemeinschaften usw.

Ein B-Web vom Typ Allianz weist folgende Vorteile auf:

- Netzwerkbildung: Partner einer Allianz bilden ein Netz von Gleichberechtigten.

- Selbstorganisation: Wenige Verhaltensregeln dienen der Zusammenarbeit.

- Prosumer: Die Partner sind gleichzeitig Producer (Hersteller von kreativen Produkten und Dienstleistungen) und Consumer (Nachfrager von Lösungen).

- Wertschöpfungsraum: Immaterielle Werte werden gemeinsam geschaffen.

- Idealisierte Zielsetzung: Gegenseitiger Respekt, Vertrauen und gemeinsame Wertschöpfung bilden die Basis der Allianz.

Eine Allianz ist ein virtuelles Netzwerk für kreative Lösungen, das auf hierarchische Führungsstrukturen verzichtet. Die Teilnehmer einer Allianz bilden eine schöpferische Gemeinschaft, die auf ein Ziel ausgerichtet ist. Sie bleibt damit eine der vergänglichsten und gleichzeitig innovativsten Form eines Business Web.
Als Beispiel eines Business Web vom Typ Allianz kann die Entwicklung des Betriebssystems Linux angesehen werden. Nachdem ein einfacher Kern eines Unix-Clons für PCs entwickelt war, wurde dieser im Internet für Weiterentwicklungen (Open Source) freigegeben. Die Nutzer sind verpflichtet, das Softwareprogramm und mögliche Erweiterungen mit dem Quellcode weiterzugeben. Dadurch entstand ein umfangreiches Betriebssystem für unterschiedliche Zwecke.

Die Form des Business Web Allianz findet im Sinne eines virtuellen Unternehmensnetzwerks ihre Verwirklichung in unterschiedlichen Branchen. Das Fraunhofer IAO hat mehrere Netzwerke dieser Form in der Begründung und Identifikation von Geschäftsideen sowie in den Phasen der Partnerakquisition und der Netzwerkkonsolidierung begleitet. Eines dieser Beispiele ist als Netzwerk im Bereich unternehmensbezogener Dienstleistungen im Großraum Stuttgart tätig [Hofmann et al. 2002]. Es ist ein loses Kooperationsnetzwerk, das neue Formen der Unternehmensberatung auf einem individualisierten Level anbietet. Der Initiator des Netzwerkes war fasziniert von der Idee, nicht mehr abhängig Beschäftigte anleiten zu müssen, sondern in einem Netzwerk selbstständiger Unternehmer je nach Kundenwunsch zugeschnittene Dienstleistungen anbieten zu können.

Als Herausforderung in diesen Netzwerken lässt sich jedoch häufig Folgendes feststellen: Nicht alle Allianzteilnehmer geben gleich viel in das Netzwerk an Vorleistungen hinein, sondern nehmen mehr, als sie investieren. Oft stellt sich heraus, dass Vorleistungen bzw. netzwerkbezogene Leistungen quantifiziert bewertet und zum Ausgleich gebracht werden müssen. Auch fällt es vielen Netzwerkpartnern schwer, bestehende Kontakte einzubringen, da sie mit dem eigenen Unternehmenserfolg verknüpft sind; besser ist es, gemeinsam Neukunden anzugehen. Ein stetes Thema ist die Frage, wie stark bei Kundenkontakten die individuelle Corporate 
Identity im Vordergrund steht oder eine virtuelle Identity erschaffen werden muss. Stark unterschätzt werden oft die rechtlichen Verpflichtungen, die die Netzwerkpartner eingehen, falls das Netzwerk keine explizite eigene Rechtsform hat. Umgekehrt können Allianzen erfolgreich sein, wenn die Rollen der Partner klar und komplementär definiert werden, die Beiträge ins Netzwerk adäquate Verrechnung finden, die überwiegend elektronische Kommunikation erfolgreich gemeistert wird [Bullinger \& Hofmann 2002] und ein Management der internen und externen Beziehungen vorangetrieben wird.

\section{Business Web vom Typ Distributor}

Unter einem Distributor versteht man als B-Web ein Verteilungsnetzwerk, das materielle und immaterielle Produkte und Dienstleistungen vom Anbieter zum Nutzer bringt. Distributoren erfüllen eine Distributionsfunktion, sei es als Transportunternehmer, Stromanbieter, Finanzdienstleister, Kurier- und Postdienst, Kommunikationsnetzbetreiber oder Logistikun-

Integratoren und Allianzen leisten ihren Beitrag zur Wertschöpfung, indem sie Rohstoffe oder Ideen zu Produkten oder Dienstleistungen veredeln. Agoren und Aggregatoren selektieren Waren, bieten sie an und verhanternehmen.

deln die Preiskonditionen. Distributoren hingegen bedienen in ihrer Ursprungsform die vier bereits diskutierten B-Webs, indem sie den Austausch von Informationen, Waren und Dienstleistungen gewährleisten.

In der Abbildung 5 ist die Grundform eines B-Web vom Typ Distributor aufgezeigt. Das Distributionsnetzwerk verbindet die Hersteller von Produkten und Dienstleistungen mit den Abnehmern oder Kunden. Dabei kann es sich um physische oder digitale Netzwerke und Verteilsysteme handeln.

Ein besonderer Fall eines digitalen Kommunikationsanbieters ist der Infomediär. Darunter wird ein Vermittler verstanden, der die Informationen von Kunden sammelt, verwaltet und weiterleitet. Private Verbraucher und Unternehmen als Käufer von Waren und Dienstleistungen beanspruchen Infomediäre. Diese bieten neben der Verwaltungs- und Verteilfunktion auch Servicedienstleistungen an unter der Berücksichtigung des Datenschutzes. Sie ermöglichen den Verbrauchern resp. den übrigen B-Webs folgende Dienste:

- Management der Zugangsfunktion (Benutzeridentifikation, Autorisierung, Berechtigung) zu digitalen Kommunikationsnetzen

- Aufbau, Verwaltung und Darstellung von Eckwerten unterschiedlicher Transaktionsdaten

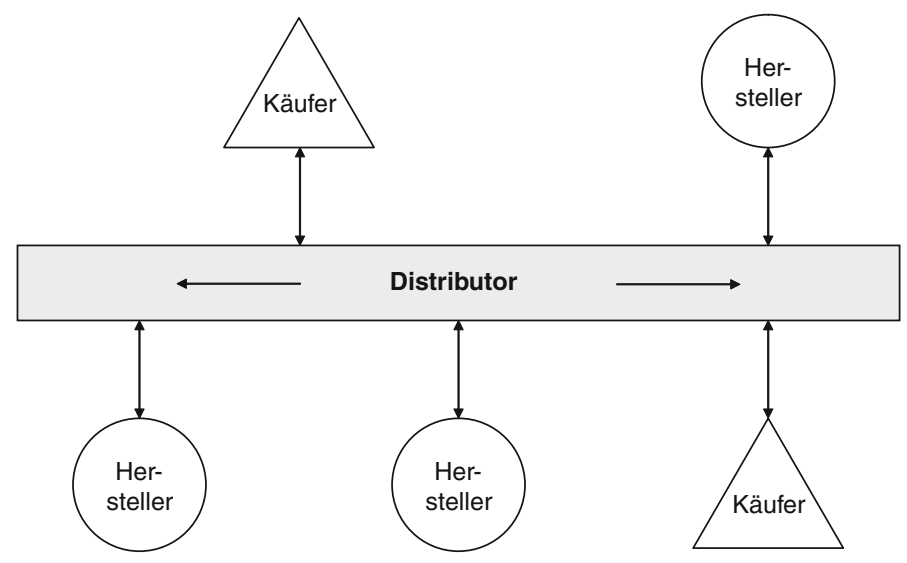

Abb. 5: Grundkonzeption des B-Web-Typs Distributor 
- Pflege von Profilen für Präferenzen in verschiedenen Themenbereichen wie Gesundheit, Unterhaltung, Kulturangebote etc.

- Darstellung des Verhaltens von Kunden und Kundengruppen als Käufer von Waren und Dienstleistungen

Infomediäre und Distributoren sind darauf spezialisiert, den Unternehmen und Privatpersonen Netzwerkdienste mit abgestuftem Leistungsdurchsatz und unterschiedlichen Sicherheitsniveaus anzubieten. Internetbasierte Distributoren versuchen, auf Ereignisse in Echtzeit zu reagieren und optimierte Distributionsprozesse anzubieten.

Die Vorteile eines B-Web vom Typ Distributor lauten:

- Internationalisierung: Der Distributor erfüllt seine Verteilungsfunktion in globalen Teilmärkten.

- Skaleneffekte: Durch die Bündelung der Transport- und Verteilfunktion können Skaleneffekte erzielt werden.

- 24-Stundenbetrieb: Viele Distributoren, vor allem bei digitalen Kommunikationslieferanten, bieten ihre Dienste täglich rund um die Uhr an.

- Spezialisierung pro Warentyp: Distributoren wirken als Netzwerkdienstleister für teilbare, weitergeleitete oder nutzbare Waren.

- Spezialfunktion des Infomediärs: Ein Infomediär steuert die Zugangsfunktion und bereitet die Transaktionsdaten auf.

Das Nutzenversprechen eines Distributors ermöglicht Austausch und Lieferung von Informationen, Waren und Dienstleistungen. Distributionsnetze tragen zur Umwandlung regulierter Monopole in wettbewerbsfähige, disaggregierte Märkte bei.

Als Beispiele von Distributoren können Telekomunternehmen und Internetprovider angesehen werden. Durch digitale Technologien entwickeln sich solche Unternehmen mehr und mehr zu Infomediären (Intermediäre in Market Space) mit entsprechenden Dienstleistungen.
Solche Distributoren können drei Angebotsformen unterstützen, abhängig von den zu transportierenden Waren:

- Netzwerkdienstleister für teilbare Waren: Sie sammeln und verteilen teilbare Güter wie Strom oder Bandbreiten für den Transport von Multimediaobjekten über digitale Kommunikationsnetze.

- Netzwerkdienstleister für weitergeleitete Waren: Dazu zählen Spediteure, Postdienste, Fluggesellschaften, aber auch Sprach-, Bildund Tonvermittler bei digitalen Kommunikationsnetzen.

- Netzwerkdienstleister für nutzbare Waren: Darunter fallen Finanz- und Versicherungsunternehmen, die Kapital verleihen oder für Risiken bürgen.

\section{Vergleich und Bewertung von Netzwerken}

Bei der Entwicklung eines Geschäftsmodells stellen sich zwei Grundsatzfragen: Welches ist das Erfolg versprechende B-Web für das Kerngeschäft des eigenen Unternehmens, und mit welchen B-Webs sollen Ergänzungsleistungen angeboten werden?

Die Typisierung von B-Webs zeigt vor allem die grundlegenden Merkmale und die entsprechenden Nutzungsaspekte auf; einen Vergleich dazu gibt Abbildung 6 .

Zielsetzung und Hauptmerkmale der BWebs sind recht unterschiedlich und reichen von freien Marktplätzen über straff organisierte Netzwerke bis zu selbstorganisierten und lose gekoppelten Gemeinschaften.

Die Rolle der Kunden in den unterschiedlichen B-Webs unterscheidet sich ebenfalls. Bei der Agora ist der Kunde Marktteilnehmer, der sich am Marktgeschehen orientiert und den Austausch von Waren verhandelt. Die Käuferrolle des Kunden beim Aggregator resp. die Empfängerrolle beim Distributor wird erweitert beim Integrator, indem hier der Kunde zu einem Wertmotor aufsteigt. Eine Allianz funk- 


\begin{tabular}{|c|c|c|c|c|c|}
\hline & Agora & Aggregator & Integrator & Allianz & Distributor \\
\hline $\begin{array}{l}\text { Ziel- } \\
\text { setzung }\end{array}$ & $\begin{array}{l}\text { Marktplatz für } \\
\text { Waren und Werte }\end{array}$ & $\begin{array}{l}\text { digitaler } \\
\text { Supermarkt }\end{array}$ & $\begin{array}{l}\text { optimierte } \\
\text { Wertschöpfungs- } \\
\text { kette }\end{array}$ & $\begin{array}{l}\text { selbstorgani- } \\
\text { sierender } \\
\text { Wertschöpfungs- } \\
\text { raum }\end{array}$ & $\begin{array}{l}\text { Austausch von } \\
\text { Informationen. } \\
\text { Waren und } \\
\text { Diensten }\end{array}$ \\
\hline Merkmale & $\begin{array}{l}\text { - Marktinfor- } \\
\text { mation } \\
\text { - Verhandlungs- } \\
\text { prozess } \\
\text { - dynamische } \\
\text { Preisfindung }\end{array}$ & $\begin{array}{l}\text { - Auslage von } \\
\text { Produkten } \\
\text { - fester Preis } \\
\text { - einfache } \\
\text { Erfüllung }\end{array}$ & $\begin{array}{l}\text { - gezielte } \\
\text { Lieferanten- } \\
\text { auswahl } \\
\text { - Prozess- } \\
\text { optimierung } \\
\text { - Produkt- } \\
\text { integration }\end{array}$ & $\begin{array}{l}\text { - Innovation } \\
\text { - Vertrauens- } \\
\text { bildung } \\
\text { - Verzicht auf } \\
\text { hierarchische } \\
\text { Kontrolle }\end{array}$ & $\begin{array}{l}\text { - Netz- } \\
\text { optimierung } \\
\text { - uneinge- } \\
\text { schränkte } \\
\text { Nutzung } \\
\text { - Logistik- } \\
\text { prozess }\end{array}$ \\
\hline $\begin{array}{l}\text { Kunden- } \\
\text { rolle }\end{array}$ & Marktteilnehmer & Käufer & Wertmotor & Beitragender & Empfänger \\
\hline Nutzen & $\begin{array}{l}\text { verhandelbare } \\
\text { Marktleistung }\end{array}$ & $\begin{array}{l}\text { bequeme } \\
\text { Auswahl und } \\
\text { Erfüllung }\end{array}$ & $\begin{array}{l}\text { kunden- } \\
\text { spezifisches } \\
\text { Produkt }\end{array}$ & $\begin{array}{l}\text { kreative und } \\
\text { gemeinschaftliche } \\
\text { Lösung }\end{array}$ & $\begin{array}{l}\text { zeitgerechte } \\
\text { Lieferung }\end{array}$ \\
\hline Beispiele & $\begin{array}{l}\text { eBay } \\
\text { auctions.yahoo }\end{array}$ & $\begin{array}{l}\text { etrade } \\
\text { amazon }\end{array}$ & $\begin{array}{l}\text { Cisco } \\
\text { Dell }\end{array}$ & $\begin{array}{l}\text { Linux } \\
\text { Music.download }\end{array}$ & $\begin{array}{l}\text { UPS } \\
\text { AT\&T } \\
\text { Telekom }\end{array}$ \\
\hline
\end{tabular}

Abb. 6: Hauptmerkmale der unterschiedlichen B-Webs im Vergleich

tioniert nur, wenn der Kunde sich einbringt und an der Lösungssuche und -entwicklung mitwirkt.

In konkreten elektronischen Märkten treten die Marktteilnehmer nicht immer als klar abgrenzbare B-Webs auf. Vielmehr weist ein bestimmtes B-Web oft Eigenschaften eines anderen B-Web auf. Zudem lassen sich verschiedene B-Webs in einem eigenen Netz von Anbietern und Kunden integrieren. Des Weiteren liegt es auf der Hand, dass jedes B-Web eine Distributionsfunktion aufweisen muss, eventuell ausgelagert bei einem marktgängigen Distributor.

\section{Ausblick}

Geschäftsmodelle befinden sich in Bewegung, und jede technologische Innovation ruft Änderungen und Anpassungen sowie neue Möglichkeiten hervor (vgl. Geschäftsmodelle im Web 2.0 von [Wirtz \& Ullrich 2008]).
Wir fassen die drei wesentlichen Stoßrichtungen der Realisierung von Business Webs in elektronischen Märkten zusammen:

- Wertschöpfungskonfigurationen werden erweitert, indem der Kunde dazu gebracht wird, vermehrt Inhalte z. B. in Form von Bewertungen einzustellen und damit neue "Währungen« zu generieren.

- Eine weitere Stoßrichtung ist das Web Branding, wie z. B. die Erhöhung des eigenen Markenwertes durch größere Bekanntheit.

- Ertragsmodelle werden differenzierter, wie z. B. durch die Realisierung von zusätzlichen Einnahmequellen durch Cross-Referenzierung oder individualisiertes Marketing.

Speziell durch die mit Web 2.o offerierten Möglichkeiten der Content-Einstellung, der Kommunikation und des Austausches der Nutzer werden klassische Anbieter- und Abnehmerschnittstellen stark verändert. Die Entwicklungsdynamik mit möglichen Rückschlägen bei 
den einzelnen Anbietern bleibt groß. So zeigen die aktuellen Entwicklungen im Kontext der Schwierigkeiten der Betreiber des Netzwerkes XING oder von studiVZ, dass die Machbarkeit neuer Erlösmodelle z. B. durch individualisierte Werbung nicht an technischen Schwierigkeiten, sondern an Widerständen der Nutzergruppen scheitern kann. Der Abgleich zwischen technisch möglichen, organisatorisch machbaren und marktseitig akzeptierten Lösungen bleibt ein dauerhaft auszutarierendes Kräftefeld.

\section{Literatur}

[Bliemel et al. 2000] Bliemel, F.; Fassott, G.; Theobald, A. (Hrsg.): Electronic Commerce - Herausforderungen, Anwendungen, Perspektiven. Gabler, 2000.

[Bullinger 1998] Bullinger, H.-.: Neue Wettbewerbsparadigmen für die Textil- und Bekleidungsindustrie. In: IAO-Forum: Accurate Response Manufacturing für die Textil- und Bekleidungsindustrie, unveröffentlichte Veranstaltungsdokumentation, 1998.

[Bullinger \& Berres 2000] Bullinger, H.-.; Berres, A. (Hrsg.): E-Business - Handbuch für den Mittelstand - Grundlagen, Rezepte, Praxisberichte. Springer-Verlag, Heidelberg, 2000.

[Bullinger \& Hofmann 2002] Bullinger, H.-J.; Hofmann, J.: Medienkompetenz: zur erfolgreichen Zusammenarbeit in e-Teams. In: Spoun, S.; Wunderlich, W. (Hrsg.): Medienkultur im digitalen Wandel. Bern, 2002.

[Hofmann et al. 2002] Hofmann, J. et al.: Besser arbeiten in Netzwerken - Wie virtuelle Unternehmen Erfolg haben. Aachen, 2002.

[Götze 1995] Götze, O.: Aufbau und Betrieb eines Online-Handels unter Berücksichtigung betriebswirtschaftlicher Aspekte anhand eines praktischen Beispieles. Diplomarbeit Hamburg, 1995.

[Meier 2001] Meier, A. (Hrsg.): Internet \& Electronic Business - Herausforderung an das Management. Orell Füssli, 2001.

[Meier \& Stormer 2005] Meier, A.; Stormer, H.: eBusiness \& eCommerce? Management der digitalen Wertschöpfungskette. Springer-Verlag, Heidelberg, 2005.
[Peters \& Reitzenstein 2008] Peters, R.; Reitzenstein, l.: Reputationssysteme im eCommerce Funktionsweise, Anwendung und Nutzenpotenziale. In: HMD - Praxis der Wirtschaftsinformatik, Heft 261, 2008, S. 43.

[Schlögel et al. 2002] Schögel, M.; Tomczak, T.; Belz, C. (Hrsg.): Roadm@p to E-Business - Wie Unternehmen das Internet erfolgreich nutzen. Verlag Thexis, 2002.

[Shapiro \& Varian 1999] Shapiro, C.; Varian, H. R.: Information Rules - A Strategic Guide to the Network Economy. Harvard Business School Press, 1999.

[Shaw et al. 2000] Shaw, M.; Blanning, R.; Strader, T.; Whinston, A. (Eds.): Handbook on Electronic Commerce. Springer-Verlag, Heidelberg, 2000.

[Tapscott et al. 2001] Tapscott, D.; Ticoll, D.; Lowy, A. Digital Capital ? Von den erfolgreichen Geschäftsmodellen profitieren. Campus Verlag, 2001.

[Timmers 1999] Timmers, P.: Electronic Commerce Strategies and Models for Business-to-Business Trading. Wiley, 1999.

[Wirtz 2000] Wirtz, B. W.: Electronic Business. Gabler, 2000.

[Wirtz \& Ullrich 2008] Wirtz, B. W.; Ullrich, S.: Geschäftsmodelle im Web 2.0 ? Erscheinungsformen, Ausgestaltung und Erfolgsfaktoren. In: HMD - Praxis der Wirtschaftsinformatik, Heft 261, 2008, S. 20.

Prof. Dr. Andreas Meier Universität Fribourg Department of Informatics Bd de Pérolles 90 $\mathrm{CH}-1700$ Fribourg andreas.meier@unifr.ch www.diuf.unifr.ch/is

Dr. Josephine Hofmann Competence Center Business Performance Fraunhofer-Institut für Arbeitswirtschaft und Organisation Nobelstr. 12 70569 Stuttgart josephine.hofmann@iao.fraunhofer.de www.businessmanagement.iao.fhg.de 